tissue $\mathrm{Cu}, \mathrm{Fe}$, and $\mathrm{Zn}$ as main determinant in experimental tetraethyllead encephalopathy. Life Sci., 13: 897 (1973).

24. Packer, L., and Jacobs, E. E.: Coupling of phosphorylation to terminal segments of the mitochondrial respiratory chain. Biochim. Biphys. Acta, 57: 371 (1962).

25. Patel, A. J., Michaelson, I. A., Cremer, J. E., and Balázs, R.: The metabolism of $\left[{ }^{14} \mathrm{C}\right]$ glucose by the brains of suckling rats intoxicated with inorganic lead. $\mathrm{J}$. Neurochem., 22: 581 (1974)

26. Patel, A. J., Michaelson, I. A., Cremer, J. E., and Balázs, R.: Changes within metabolic compartments in the brains of young rats ingesting lead. J Neurochem., 22: 591 (1974).

27. Pentschew, A., and Garro, F.: Lead encephalomyelopathy of the suckling rat and its implications on the porphyrinopathic nervous diseases. Acta Neuropathol., 6: 266 (1966)

28. Potter, V. R., Schneider, W. C., and Liebl, G. J.: Enzyme changes during growth and differentiation in the tissues of the newborn rat. Cancer Res., 5: 21 (1945).

29. Rhyne, B. C., and Goyer, R. A.: Cytochrome content of kidney mitochondria in experimental lead poisoning. Exp. Mol. Pathol., 14: 386 (1971).

30. Sanadi, D. R., and Jacobs, E. E.: Assay of oxidative phosphorylation at the cytochrome oxidase region (site III). 10:38 (1967).

31. Scott, K. M., Hwang, K. M., Jurkowitz, M., and Brierly, G. P.: Ion transport by heart mitochondria. XXIII. The effects of lead on mitochondrial reactions.

Copyright (c) 1976 International Pediatric Research Foundation, Inc.
Arch. Biochem. Biophys., 147: 557 (1971)

32. Thomas, J. A., and Thomas, I. M.: The pathogenesis of lead encephalopathy. Indian J. Med. Res., 62: 36 (1974).

33. Tyler, D. B., and Van Harreveld, A.: The respiration of the developing brain. Amer. J. Physiol., 136: 600 (1942).

34. Ralston Purina Co., St. Louis, Mo.

35. St. Louis, Mo.

36. Kanakee, III.

37. Phillipsburg, N. J.

38. Rochester, N. Y

39. San Diego, Calif

40. Ms. Emily Brewer is thanked for her technical assistance.

41. Dr. D. Holtzman is a Mellon Foundation Fellow.

42. These results were presented, in part, at the Sixth Meeting of the American Society for Neurochemistry.

43. This investigation was supported by a grant from the Research Development Fund of Stanford University.

44. Requests for reprints should be addressed to: D. Holtzman, M.D., Ph.D., Department of Neurology, Stanford University Medical Center, Stanford, Calif. 94305 (USA).

45. Accepted for publication September 16, 1975.

Printed in U.S.A.

Pediat. Res. 10: 75-78 (1976)

Cell-mediated immunity

inactivated vaccine lymphocyte transformation respiratory syncytial virus

\title{
Cell-mediated Immunity to Respiratory Syncytial Virus Induced by Inactivated Vaccine or by Infection
}

\author{
HYUN WHA KIM, SANFORD L. LEIKIN, JULITA ARROBIO, CARL D. BRANDT, \\ ROBERT M. CHANOCK, AND ROBERT H. PARROTT ${ }^{(13)}$ \\ George Washington University, Research Foundation of Children's Hospital Medical Center, Washington, D.C. and \\ Laboratory of Infectious Diseases, National Institute of Allergy and Infectious Diseases, National Institutes of \\ Health, Bethesda, Maryland, USA
}

\section{Extract}

Transformation and increased mitotic activity in donor lymphocytes exposed to specific antigens is considered by many to be a manifestation of cell-mediated immunity. In attempts to understand the apparent "sensitization" of individuals to respiratory syncytial virus (RSV) as a result of receiving inactivated RSV vaccine, in vitro lymphocyte transformation studies were carried out on infants who had received inactivated RSV vaccine and on infants who had received a similarly prepared inactivated African green monkey kidney (AGMK) cell-grown parainfluenza type 1 virus vaccine or a trivalent parainfluenza vaccine prepared in hen's eggs. Each group included some infants who had, and others who had not, undergone natural RSV infection under our observation before the lymphocyte studies.

Lymphocytes were studied from 21 infants and young children who had received the inactivated $\mathrm{RSV}$ vaccine, 14 who received a similarly prepared inactivated parainfluenza 1 vaccine, and 5 who received a trivalent parainfluenza vaccine. Twelve of the RSV vaccinees and 14 of the parainfluenza vaccinees had been naturally infected with RSV as indicated by virus recovery and/or antibody rise between the time of vaccination and the Iymphocyte studies. In comparing the arithmetic mean for RSV-specific transformation and mitotic activity there was a significant difference between RSV vaccinees and parainfluenza vaccinees whether one compared those who had undergone natural RSV infection or those who had not undergone natural infection. The difference between $\mathrm{RSV}$ vaccinees who had not undergone natural RSV infection and RSV-infected parainfluenza vaccinees also was significant. There was a greater level of transformation and mitotic activity in those who had experienced natural infection than those who had not among both RSV vaccinees and parainfluenza vaccinees, but these differences were not significant statistically.

\section{Speculation}

We take these findings to mean that natural RSV infection probably stimulates a systemic cell-mediated immunity response and that such a response is definitely induced after administration of killed RSV antigen. These findings are consistent with the hypothesis that cell-mediated sensitization may in some way contribute to the altered response to natural infection which occurred after use of inactivated RSV vaccine. Our findings do not support the hypothesis that systemic cell-mediated immunity per se is important in protecting against RSV infection. The findings also suggest the possibility that transplacentally conferred RSV lymphocyte sensitization might play a part in the pathogenesis of nonvaccine related RSV bronchiolitis which characteristically occurs during early infancy, frequently in the presence of measurable serum antibody.

The first potential vaccines against RSV in infants were inactivated virus preparations. One of these was a 100 times concentrated virus which had been grown in AGMK cell cultures 
(7). This vaccine induced significant serum antibody levels when administered to infants; however, infants who received this preparation were not protected against infection or illness at the time of subsequent natural exposure to RSV. Furthermore, RSV illness in these individuals was more severe than that in a control group who had received an AGMK-grown inactivated parainfluenza type 1 virus vaccine. In many instances, RSV vaccinees developed serious illness during the second year of life in a form simulating RSV bronchiolitis of young infants. These observations indicated that RSV-specific antigens present in the inactivated vaccine induced a state of altered reactivity ot RSV infection.

Transformation and mitotic activity in donor lymphocytes exposed to specific antigens are considered by many to be manifestations of cell-mediated immunity. In attempts to understand the apparent sensitization of individuals to RSV as a result of receiving inactivated RSV vaccine, in vitro lymphocyte transformation studies were carried out on infants who had received inactivated RSV vaccine as well as on infants who had received a similarly prepared inactivated AGMK cell-grown parainfluenza type 1 virus vaccine or a trivalent parainfluenza vaccine prepared in hen's eggs. Each group included some infants who had, and others who had not, undergone natural RSV infection under our observation before the lymphocyte studies.

Findings suggesting that RSV-specific systemic cell-mediated immunity develops after immunization with killed RSV vaccine and probably after natural RSV infection are reported here with comments and questions on the implications of these findings for understanding the pathogenesis and prevention of RSV disease.

\section{METHODS}

\section{CLINICAL}

Lymphocytes from forty infants and young children were studied during 1967 after the disease potentiation induced by the RSV vaccine was first recognized. Included were 21 individuals who had received 100 times concentrated inactivated RSV vaccine prepared in AGMK cells, 14 who had received a 100 times concentrated inactivated parainfluenza 1 vaccine also prepared in AGMK cells, and 5 who had received an inactivated trivalent parainfluenza vaccine prepared in hen's eggs. The lymphocyte studies were carried out 2-14 months after vaccine was administered (7). All infants and children in this report participated in the study with the specific written consent of their parents or guardians.

The RSV and parainfluenza vaccinees were kept under surveillance and sera were collected at least three times a year. Also an attempt was made to recover RSV at the time of each acute respiratory illness. Between the time of vaccination and the lymphocyte studies 12 of the 21 RSV vaccinees and 14 of the 19 parainfluenza vaccinees were infected with RSV as indicated by virus recovery and/or a fourfold or greater rise in serum plaquereduction antibody. Two adults were studied to provide a comparison group for the infants and in order to evaluate the methods used.

\section{LYMPHOCYTE TRANSFORMATION STUDIES}

Blood for lymphocyte transformation studies was drawn into heparinized syringes. The erythrocytes were allowed to sediment for 30-60 min at $37^{\circ}$ and the leukocyte-rich plasma was collected into sterile test tubes. Total white blood cell and differential cell counts were performed. Then $5 \times 10^{6}$ mononuclear cells were inoculated into $5 \mathrm{ml}$ tissue culture fluid consisting of medium NCTC 109 and 20\% autologous plasma. Five cultures were prepared with lymphocytes from each individual. A stimulant was omitted from one tube which served as control. Two cultures were stimulated with an AGMK preparation of live RSV and two were stimulated with an AGMK cell control preparation. Both types of stimulant preparations were prepared by zonal centrifugation in a sucrose gradient and represented a 100 -fold concentration of the original AGMK suspension (4). Two dilutions of each stimulant were used (1:10 and 1:100); the $1: 10$ dilution usually gave a higher percentage of blast transformation. For purposes of tabulation and analysis the higher of the two values obtained with the RSV preparation and the value obtained with the corresponding dilution of uninfected AGMK were used.

Lymphocyte cultures were incubated at $37^{\circ}$ for 7 days, after which they were centrifuged at $800 \mathrm{rpm}$ for $5 \mathrm{~min}$ and smears made. The smears were stained with Wright-Giemsa, dried, and mounted. The percentage of lymphocyte transformation and mitotic activity in these cultures and in unstimulated control cultures was estimated by microscopic examination of 1,000 cells in each preparation.

The percentage of lymphocytes responding to specific antigen was recorded as observed but should be interpreted as only semiquantitative. This is because sensitized cells can respond with the production of specific mitogens which then may "recruit" additional lymphocytes to undergo transformation.

\section{RESULTS}

The response of lymphocytes to AGMK and AGMK-grown RSV is analyzed by $(l)$ vaccine group and (2) whether infection was detected after immunization but before the time of testing (Table 1). All of the RSV vaccinees, whether or not they had been infected subsequently with the virus, showed lymphocyte transformation and mitotic activity in response to RSV antigen which was at least $2 \%$ greater than was observed with lymphocytes exposed to uninfected AGMK. Nine of 14 infants who had received a parainfluenza vaccine and who had undergone natural RSV infection under our observation exhibited a response; however, their lymphocytes showed a lower level of transformation than did lymphocytes from individuals who had received the RSV vaccine. Significantly, only one of five parainfluenza vaccinees who did not appear to have undergone RSV infection showed an excess of transformation in response to RSV antigen over that seen with uninfected AGMK, and this was at a low level (1.7\%). Lymphocyte transformation activity also seemed to correlate with the presence of serum complement-fixing antibody against RSV.

Another, possibly more valid, way to evaluate differences among these groups is to compare the arithmetic mean of transformation and mitotic activity for each group. By this method, there was a significant difference between RSV vaccinees and parainfluenza vaccinees whether one compared those who had undergone natural RSV infection $(P<0.001)$ or those who had not undergone natural infection $(P<0.02)$. The difference between RSV vaccinees who had not undergone natural RSV infection and parainfluenza vaccinees who had was also significant $(P<0.01)$. Finally, there was a greater level of transformation and mitotic activity exhibited by parainfluenza vaccinees who had undergone natural RSV infection than those who had not; however, this difference was not significant statistically.

\section{DISCUSSION}

The findings in this study indicate that lymphocytes of individuals who had undergone natural RSV infection and/or had received alum-precipitated inactivated RSV vaccine were conditioned to respond to RSV antigen. The response was specific to RSV in that infants who received a parainfluenza vaccine did not show significant lymphocyte sensitivity unless they had evidence of having undergone natural RSV infection. The RSV-specific response after administration of vaccine was clearly statistically significant and the level of activity in RSV vaccinees was definitely higher than in individuals who had received a parainfluenza vaccine and who underwent natural RSV infection. The findings also suggest that natural infection produced RSV-sensitized lymphocytes but there were too few uninfected individuals to provide sufficient data to make for statistical significance.

Although these studies were performed just before the develop- 
Table 1. Transformation and mitotic activity of lymphocytes from infants who had received inactivated 100 times concentrated respiratory syncytial virus ( $R S Y$ ) vaccine or parainfluenza vaccines ${ }^{1}$

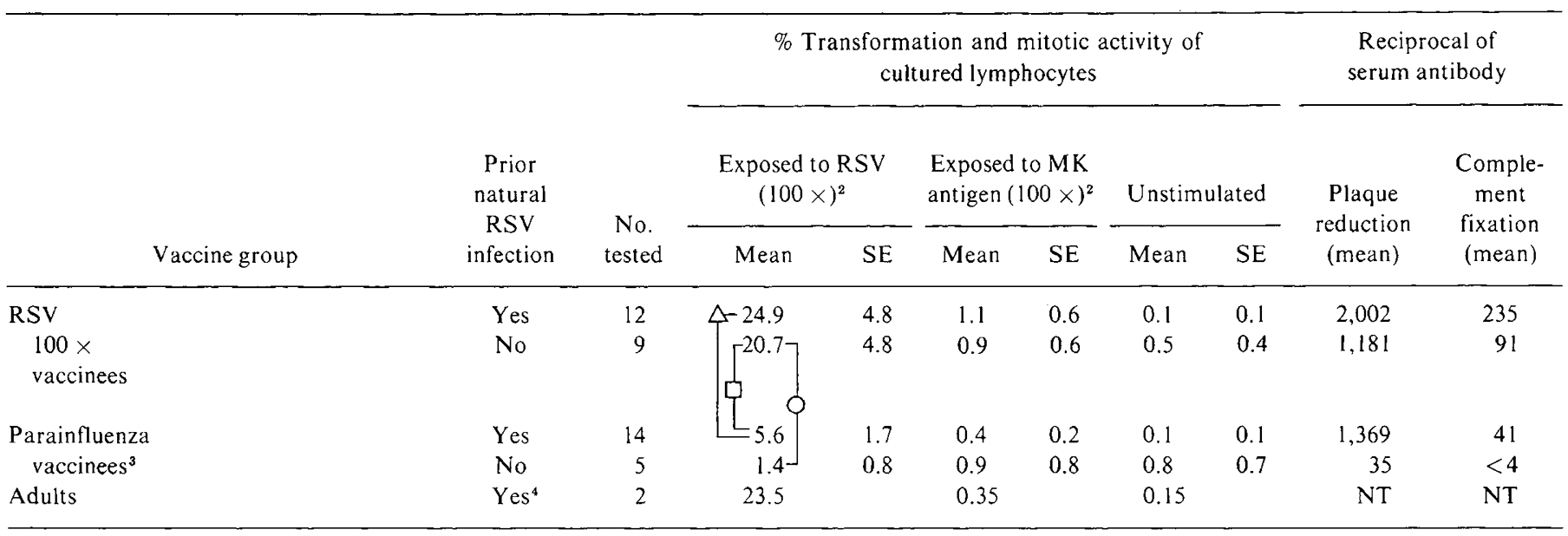

${ }^{1}$ Two dilutions (1:10 and 1:100) of stimulant were used; the values included in the table represent the higher of the two responses observed. In each instance the response to the corresponding dilution of uninfected monkey kidney (MK) antigen was included in the analysis. $\triangle$ : significantly different, Student's $t$-test; $P<0.001$; O: significantly different, Student's $t$-test; $P<0.02 ; \square$ : significantly different, Student's $t$-test; $P<0.01$; NT: not tested.

${ }^{2}$ Prepared by zonal centrifugation in sucrose gradient.

${ }^{3}$ Fourteen received a 100 times concentrated inactivated parainfluenza 1 vaccine which had been grown in MK cell cultures; five others received unconcentrated trivalent parainfluenza 1,2 , and 3 vaccine prepared in embryonated hen's eggs.

${ }^{4}$ One was tested for neutralizing antibody, the other is assumed to have undergone natural infection.

ment of more sophisticated methods (such as radioactive thymidine labeling) to measure lymphocyte response, the results seem clear-cut enough for interpretation. We take them to mean that natural RSV infection stimulates systemic cell-mediated immunity (CMI) response and also that such responses can be induced by administration of killed RSV antigen. This interpretation should be taken with caution since in a number of animal species transformation may occur in " $b$ " lymphocytes as well as in " $t$ " lymphocytes. However, recent studies suggest that in man " $t$ " but not "b" lymphocytes respond to antigen by undergoing transformation (4). It should be noted that lymphocyte transformation is only a part of the full process of CMI. Mediators are presumably necessary to produce a cell-killing effect and these were not assayed.

Nonetheless, it seems likely that the transformation noted did represent systemic CMI. Because no studies were done with lymphocytes from the respiratory tract, no conclusions can be made on the very important question of whether local plus systemic CMI were present after natural infection; one might expect, however, that probably only systemic CMI was present after killed vaccine.

These findings are consistent with the hypothesis that cellmediated sensitization may in some way contribute to the altered response to natural infection which occurred after use of the inactivated RSV vaccine. It is known that infection with RSV by the respiratory route, whether natural or artificial, will induce local respiratory tract immunity in the form of local secretory antibody (4). Possibly a local CMI response also occurs. Furthermore, local immunity minimizes the extent of subsequent infection. Intramuscular vaccination, at least with an inactivated antigen, presumably would not be effective in inducing respiratory tract immunity. In the absence of this immunity, subsequent natural RSV infection would extend readily along the respiratory tract. RSV antigen would then encounter either serum antibody and/or other sensitized host immune mechanisms in the lower respiratory tract and cause a bronchiolitis-like illness. The question of the precise role of cell-mediated immunity in the "hypersensitivity" induced by the inactivated vaccine is unsettled and probably will remain so because it is unlikely that others will administer killed RSV vaccine to infants.

The present findings would also appear to negate the hypothesis that systemic cell-mediated immunity per se is important in protecting against RSV disease. All of the infants who received the RSV vaccine showed significant RSV-specific lymphocyte transformation despite the fact that some of them had been studied before, and others after their first natural infection with RSV. Elsewhere we have reported the failure of serum antibody to protect against infection and the paradoxical occurrence of an increase in serious disease in vaccinees when they encountered natural infection (7). Thus neither measurable systemic CMI nor serum antibody induced by vaccination apparently protect against RSV disease.

The pathogenesis of nonvaccine-related RSV bronchiolitis which characteristically occurs during early infancy remains unclear. The peak incidence of such illness is about 2 months of age when maternally conferred serum immunoglobulin G RSV antibody is high $(2,3)$. We have previously postulated that serum antibody in the absence of local antibody may play a part in the production of illness $(2,3)$. However, after more detailed study it is clear that some infants and children who do not possess measurable serum antibody may experience RSV bronchiolitis (10).

In view of the findings in the present paper, we now wonder if transplacentally conferred lymphocyte sensitization to RSV might play a part. There are increasing numbers of reports and speculations on the transmission of antigen-specific lymphocyte sensitivity to the fetus $(1,5,6,8,9)$. It is possible that RSV sensitized the cells themselves or that other RSV-specific material, such as transfer factor, might traverse the placenta to sensitize lymphocytes in the newborn. Lymphocytes so sensitized would be expected to decrease in numbers with age and such an occurrence would be compatible with the unique age distribution of RSV bronchiolitis. Studies to explore this possibility are under way.

\section{SUMMARY}

In an attempt to understand the apparent altered reactivity of individuals to RSV induced by inactivated RSV vaccine, in vitro lymphocyte transformation was used to study cell-mediated immunity of infants and children who had received inactivated RSV vaccine, a similar parainfluenza type 1 virus vaccine, or egg-grown trivalent parainfluenza vaccine. Some individuals in each group had undergone natural RSV infection before the lymphocyte 
studies. All RSV vaccinees, some of whom had undergone RSV infection, and most individuals who received a control parainfluenza vaccine and underwent RSV infection showed RSV-specific lymphocyte transformation. These findings strongly suggest that systemic RSV-specific, cell-mediated immunity developed after immunization with killed RSV vaccine and after natural RSV infection. As applied to understanding the pathogenesis and prevention of RSV disease these findings $(I)$ are consistent with the hypothesis that systemic cell-mediated sensitization may have contributed to the altered response to natural infection which occurred after the use of inactivated RSV vaccine; (2) are opposed to the hypothesis that systemic cell-mediated immunity per se is important in protection against RSV disease; (3) suggest the hypothesis that transplancentally conferred lymphocyte sensitization to RSV might play a part in the pathogenesis of first infection RSV bronchiolitis of early infancy.

\section{REFERENCES AND NOTES}

1. Beer, A. E., and Billingham, R. E.: Maternally acquired runt disease. Science, 179: 240 (1973).

2. Chanock, R. M., Parrott, R. H., Kapikian, A. Z, Kim, H. W and Brandt, C. D.: Possible role of immunologic factors in pathogenesis of RS virus lower respiratory tract disease. Perspect. Virol., 6: 125 (1968).

3. Chanock, R. M., Kapikian, A. Z., Mills, J., Kim, H. W., and Parrott, R. H. Influence of immunological factors in respiratory syncytial virus disease of the lower respiratory tract. Arch. Environ. Health, 21: 347 (1970).
4. Chess, L., McDermott, R. P., and Schlossman, S. S.: Immunological function of isolated human lymphocyte sub-populations. II. Antigen triggering of $\mathrm{T}$ and $\mathrm{B}$ cells. J. Immunol., 113: 1122 (1974)

5. Field, E. J., and Caspary, E. A.: Is maternal lymphocyte sensitization passed to the child? Lancet, ii: 337 (1971).

6. Gill, T. J., III: Maternal/fetal interactions and the immune response. Lancet, i: 133 (1973).

7. Kim, H. W., Canchola, J. G., Brandt, C. D., Pyles, G., Chanock, R. M., Jensen, K. E., and Parrott, R. H.: Respiratory syncytial virus disease in infants despite prior administration of antigenic inactivated vaccine. Amer. J. Epidemol., 80: 422 (1969).

8. Leikin, S., Whang-Peng, J., and Oppenheim, J. J.: In vitro transformation of human blood lymphocytes by antigens. Proceedings of the Fifth Leukocyte Culture Conference, p. 389 (Academic Press, New York. 1970).

9. Leikin, S., and Oppenheim, J. J.: Prenatal sensitization. Lancet, ii: 876 (1971).

10. Parrott, R. H., Kim, H. W., Arrobio, J. O., Hodes, D. S., Murphy, B. R., Brandt, C. D., Camargo, E., and Chanock, R. M.: Epidemiology of respiratory syncytial virus infection in Washington, D. C. II. Infection and disease with respect to age, immunologic status, race and sex. Amer. J. Epidemiol., 98: 289 (1973).

11. We would like to thank Dr. Robert Hull of the Eli Lilly Co. for supplying the concentrated RSV MKTC and normal MKTC antigens for these studies and David W. Alling, M.D., Ph.D., of the National Institutes of Health for his statistical analyses.

12. These studies were supported in part by National Institute of Allergy and Infectious Diseases Grant no. AI01528-17, Contract N1H-NIAD-71-2091, and General Clinical Research Center Grant No. 5 MO1 FR00284-05.

13. Requests for reprints should be addressed to: R. H. Parrott, M.D., Children's Hospital National Medical Center, 2125 13th St., N.W., Washington, D.C. 20009 (USA).

14. Accepted for publication September 16, 1975. 